\title{
Solubilization and Characterization of Ovomucin without Chemical Modification
}

\author{
Noboru AdachI, ${ }^{*}$ Jun-ichi Azuma, Masanobu JANADO \\ and Konoshin ONODERA \\ Laboratory of Biological Chemistry, Department of Agricultural \\ Chemistry, Kyoto University, Kyoto \\ Received May 9, 1973
}

\begin{abstract}
1. The egg white, thick and thin fractions, was solubilized in $1.0 \%$ SDS solution by vigorous mixing and subjected to gel filtration on a Sepharose $4 \mathrm{~B}$ column, eluted with $1.0 \%$ SDS. The isolated thick and thin ovomucins were found by analytical disc electrophoresis to be free from contamination with lysozyme.

2. In the velocity sedimentation the two ovomucin fractions behave similarly, both comprising at least two components with sedimentation coefficients $35 \mathrm{~S}$ and $30 \mathrm{~S}$.

3. The chemical compositions of the two ovomucin fractions showed only notable difference in that the carbohydrate content of the thick white ovomucin was somewhat higher than that of the thin white ovomucin. The amino acid profiles of the two fractions were similar.
\end{abstract}

Ovomucin, a polydisperse glycoprotein first isolated by Eichholz ${ }^{1 /}$ from egg white, is believed to be responsible for the gel-like properties of the thick white. ${ }^{2}$ Since it was shown that the inhibitory activity of egg white against hemagglutination by influenza viruses resided in the ovomucin fraction,,$^{3,4)}$ numerous attempts have been made to clarify its chemical and physical properties; and yet ovomucin is ill-defined. Undoubtedly, this is because of its poor solubility and complete insolubility after isolation. Robinson and Monsey ${ }^{5 !}$ found that ovomucin was dissolvable after reduction with 2-mercaptoethanol followed by alkylation with iodoacetic acid in $6 \mathrm{M}$ guanidinium hydrochloride. While the use of their method is limited to modified ovomucins, their finding nevertheless provided a useful means facilitating characterization of ovomucin $^{6)}$ and led to better understanding of the liquefaction mechanism of the thick white. ${ }^{7 \sim 9}$ ?

A method ${ }^{21}$ frequently employed for preparation of ovomucin involves precipitation on dilution of egg white with 4 volumes of water followed by purification of the resulting gela-

* Agricultural Development Station of Kyoto Prefecture, Ayabe. tinous precipitate by washing first with water and then with $2 \% \mathrm{KCl}$. Ovomucin thus obtained is hardly soluble in ordinary buffer solutions. Although some physicochemical properties of ovomucin have been reported by several authors, ${ }^{4,8 \sim 10)}$ it should be stressed that in these studies only soluble fractions, extractable with ordinary buffer solutions from whole ovomucin precipitate in the early stage of purification, was investigated. Robinson et al. ${ }^{11}$ ' reported that the $\mathrm{KCl}$ extract obtained during purification of crude ovomucin contained a dissolved substance that possessed the same constituent carbohydrates as ovomucin and that formed a complex with lysozyme at low ionic strength.

In order to explore the nature of native ovomucin and to demonstrate unequivocally its relation to the gel-like properties of the thick egg white, it would be essential to obtain ovomucin in soluble state. In this communication it has been shown that ovomucin is dissolvable in $0.5 \sim 1.0 \%$ SDS without chemical modification and separable in pure form by gel filtration. Also some physicochemical properties of ovomucin fraction obtained from the thick and thin white fractions were presented. 


\section{MATERIALS AND METHODS}

Materials. All reagents used were analytical reagent grade. Sodium dodecyl sulfate (SDS) was purchased from Nakarai Chemicals, Ltd., and used as supplied.

Solubilization and separation of ovomucin. Fresh egg white was divided into the thick and thin fractions by repeated decantation. Approximately $10 \mathrm{~g}$ of egg white were placed in $90 \mathrm{ml}$ of $1 \%$ SDS solution in a $300 \mathrm{ml}$ beaker and the content was mixed vigorously using a magnetic stirrer for $3 \sim 6 \mathrm{hr}$ at room temperature to solubilize completely all the egg white components. At this stage the egg white solutions were subjected to analytical gel electrophoresis to locate the ovomucin fraction among the other protein components. Noting that ovomucin is large enough to be excluded by $4 \%$ acrylamide gel, gel filtration on a Sepharose 4B column was carried out to separate ovomucin fraction, as described below.

Gel filtration. Gel chromatography was performed on a Sepharose $4 \mathrm{~B}$ column $(35 \times 2.3 \mathrm{~cm})$ equilibrated with $1 \%$ SDS solution. Approximately $10 \mathrm{ml}$ of SDS-egg white solution containing $2.6 \mathrm{mg}$ of ovomucin was placed on the column and eluted with $1 \%$ SDS solution, the flow rate being maintained at $20 \mathrm{ml} /$ hr. A fraction collector was actuated at $12 \mathrm{~min}$ interval to collect $4 \mathrm{ml}$ fractions, which were subsequently analysed for protein to produce the elution profile.

Analytical gel electrophoresis. Disc electrophoresis was carried out on $7 \%$ and $4 \%$ polyacrylamide gels in the presence of $1.0 \% \mathrm{SDS}$ in $0.1 \mathrm{M}$ sodium phosphate buffer ( $\mathrm{pH} 7.1$ ). The procedure described by Shapiro ef al. ${ }^{12)}$ was followed to prepare the gel column, and the electrophoresis was run at a constant current of $8 \mathrm{~mA} /$ column at room temperature, using bromphenol blue as the tracking dye. The gels were stained for protein with Amido Black and for carbohydrate with the periodic acid-Schiff reagent (PAS), following the procedure described by Fairbanks et al. ${ }^{13}$

Velocity sedimentation. Analytical ultracentrifugation was carried out in a Beckman model E ultracentrifuge equipped with schlieren optics. A $12 \mathrm{~mm}$ single-sector cell was used throughout. Velocity sedimentation experiments were performed at 42,040 rpm in $0.5 \%$ SDS solution, temperature being controlled at $20^{\circ} \mathrm{C}$. Apparent sedimentation coefficients were calculated from the maximum ordinate.

Chemical analysis. Concentrations of ovomucin solutions were measured by dry weight or by spectrometric measurements using the specific extinction coefficient reported by Donovan et al. ${ }^{81}$ Prior to chemical analyses, ovomucin fraction was freed of SDS by ex- haustive dialysis against $90 \%$ acetone whereby ovomucin was quantitatively precipitated. The ovomucin precipitate, after washing with acetone in a centrifuge tube, was collected by centrifugation and dried in vacuo and weighed.

Sialic acid was determined by the thiobarbituric acid method. ${ }^{14)}$ Ovomucin $(7 \sim 8 \mathrm{mg})$ was hydrolyzed with $0.025 \mathrm{M}$ sulfuric acid in a vacuum sealed tube for $1 \mathrm{hr}$ at $80^{\circ} \mathrm{C}$. N-Acetyl neuraminic acid was used as standard.

Total hexosamine was determined by the method of Cessi and Piliego ${ }^{15}$ and galactosamine by the method of Cessi and Serafini-Cessi. ${ }^{16}$ Sample $(6 \mathrm{mg})$ was hydrolyzed in $4 \mathrm{M} \mathrm{HCl}(12 \mathrm{ml})$ for $6 \mathrm{hr}$ at $100^{\circ} \mathrm{C}$ in a sealed tube.

Total hexose was estimated by the phenol-sulfuric acid method ${ }^{17 /}$ using D-glucose as standard. Since in this method sample must be in the form of solution without SDS, which interferes with the phenol-sulfuric acid procedure, analyses were made on reduced ovomucin dissolved in $0.1 \mathrm{M}$ phosphate buffer: ovomucin was reduced by 2-mercaptoethanol and alkylated as described by Robinson et al. ${ }^{6}$

The amino acid composition was analyzed using a Hitachi amino acid analyzer. Ovomucin $(10 \mathrm{mg})$ was hydrolyzed in $6 \mathrm{M} \mathrm{HCl}$ in a vacuum sealed tube for $22 \mathrm{hr}$ at $110^{\circ} \mathrm{C}$. No corrections were made for possible destruction of certain amino acids that might occur during acid hydrolysis in the presence of carbohydrate.

Protein content was obtained from the amino acid content.

\section{RESULTS}

Upon vigorous mixing of egg white $(10 \mathrm{~g})$ with 9 to 10 volumes of $1 \%$ SDS, all the egg white proteins including ovomucin were completely solubilized to give a clear solution with moderate viscosity: time required for the complete solubilization was one hour for the thin white, two hours for the thick white and five hours for chalazae. The maximum limit of egg white dissolvable in $1 \mathrm{ml}$ of SDS was approximately $0.5 \mathrm{~g}$. It may be worthy to note that the yolk membrane can be similarly dissolved by prolonged mixing $(6 \sim 20 \mathrm{hr})$.

Disc electrophoresis of the solutions of thin white, thick white and chalazae on $7 \%$ polyacrylamide gel revealed the presence of 9 welldissolved components stainable with Amido Black and at least 3 prominent carbohydratecontaining components (Fig. 1). Although the rapidly migrating component, identified 


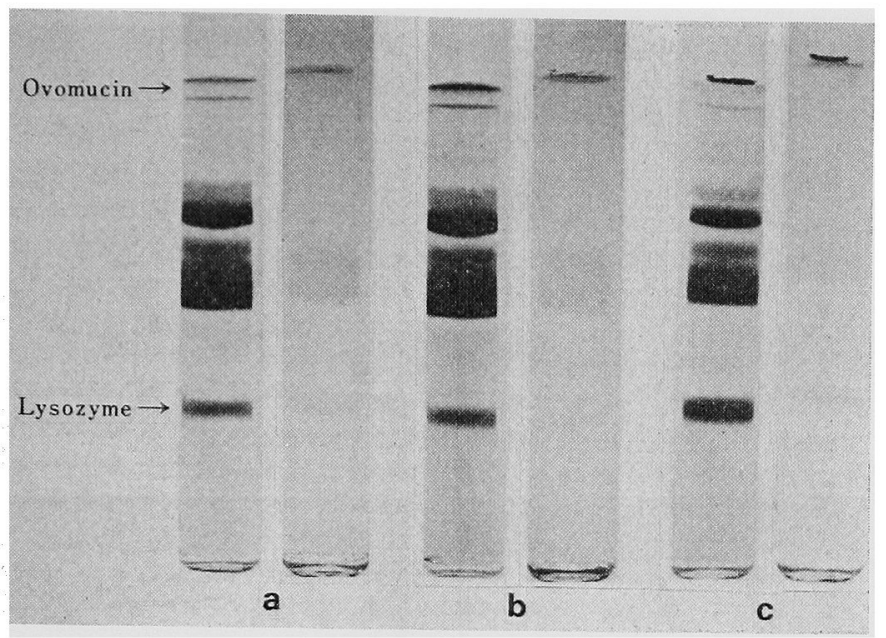

FIG. 1. Gel Electrophoresis of Thick White, Thin White and Chalazae Solubilized in $1 \%$ SDS.

Electrophoresis was performed on $7 \%$ gel in the presence of $1 \%$ SDS: a, thick white; $b$, thin white and c, chalazae; pattern on the right of each pair is stained with PAS reagent and that on the left with Amido Black.

as lysozyme by comparison of the mobility with that of an authentic sample, appears to be stained with PAS reagent, this is due to turbid appearance of lysozyme upon subjection to the PAS staining procedure. Figure 1 shows close similarity of the electrophoretic profiles of thin white, thick white and chalazae. The component accumulated at the origin was found to be ovomucin from its characteristic solubility behavior and chemical composition, as will be described later. Ovomucin was completely excluded by $4 \%$ acrylamide gel.

Separation of ovomucin from the egg whiteSDS solutions was effected by gel filtration on a Sepharose 4B column eluted with $1 \%$ or $0.5 \%$ SDS solution. The results presented in Fig. 2 show once again close similarity of the elution profiles of thick white (a) and thin white (b). Ovomucin was found in the fractions eluted at $56 \mathrm{ml}$, the void volume of the column, and apparently freed of lysozyme and other proteins as evidenced by the electrophoretic patterns jointly shown (Fig. 2). The ovomucin contents $(\mathrm{g} / 100 \mathrm{~g}$ egg white) were 0.26 for thick white and 0.18 for thin white. The fractions eluted between 68 and $88 \mathrm{ml}$ contained single component different from ovomucin in that it contained no carbohydrate and apparently migrated into $7 \%$ gel, and those

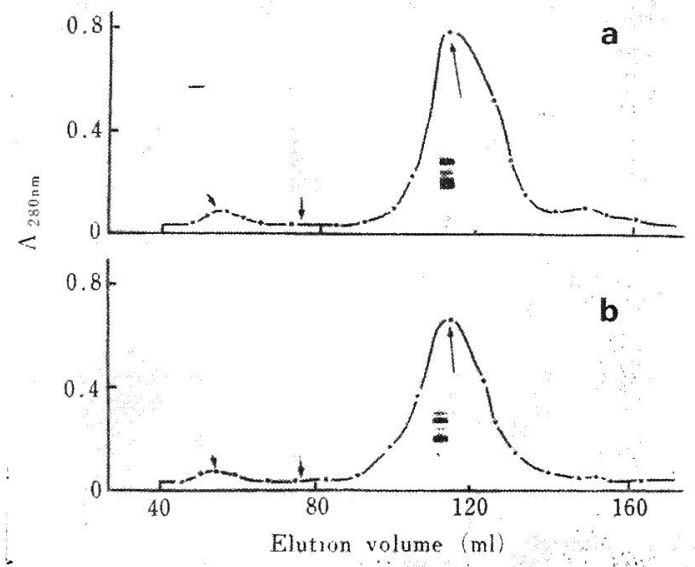

FIG. 2. Chromatographic Separation of Ovomucin from Other Protein Constituents on a Sepharose 4B Column.

Both thick and thin white were solubilized in $1 \%$ SDS; $a$, thick white and $b$, thin white; electrophoretic patterns are of the fractions respectively indicated by arrows; pattern on the right of each pair is stained with PAS reagent and on the left with Amido Black.

eluted between 96 and $130 \mathrm{ml}$, the major fraction, contained several protein components. Lysozyme is presumably contained in the fractions appearing behind the major peak which were not further investigated. In conclusion, the results (Fig. 2) shows that ovomucin can be isolated in pure form by 


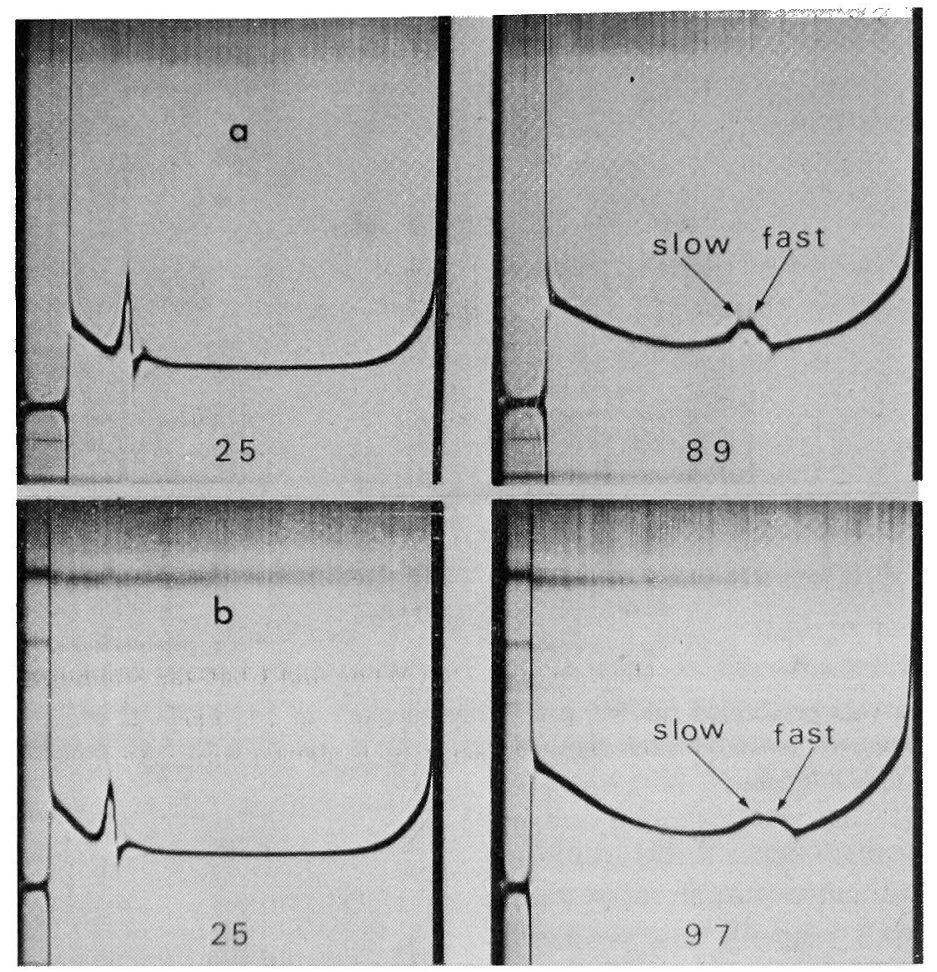

FIG. 3. Sedimentation Diagrams of the Ovomucins Isolated from Thick and Thin White Fractions.

Time the photographs taken after reaching a speed of $42,040 \mathrm{rpm}$ is indicated below the respective patterns; a (top pictures): thick white ovomucin, and b (bottom pictures): thin white ovomucin; phase plate angle was $60^{\circ}$ and concentrations of the two ovomucin fractions were $2.0 \mathrm{mg} / \mathrm{ml}$.

gel filtration on Sepharose 4B. Using a larger column $(37 \times 3.5 \mathrm{~cm})$, it was possible to obtain $5 \mathrm{mg}$ of ovomucin by one run.

Velocity sedimentation of the purified ovomucin fractions was carried out in $0.5 \%$ SDS solution to minimize density gradient formation of the back ground solution. The velocity patterns of the ovomucin fractions (thick white (a) and thin white (b) ovomucins) were shown in Fig. 3. The patterns exhibit bizarre biphasic schlieren boundaries in early stage of ultracentrifugation, but as sedimentation progressed the biphasic patterns of the two ovomucins were gradually reformed into the normal boundaries, respectively composed of two or possibly three components. Thus, again the two ovomucin fractions behave similarly in velocity sedimentation and are both heterogeneous comprising two or three components. Furthermore, sedimentation coefficients of the respective ovomucin com- ponents of thick and thin white fractions are quite similar, if not the same, as will be discussed in the following section. The bizarre biphasic pattern, which represents an extreme case of the Johnston-Ogston effect, ${ }^{18)}$ often results when a mixture comprising two or more components differing in density is subjected to velocity sedimentation. ${ }^{19,20)}$ Therefore, it appears that ovomucin is composed of two or more components differing in density or in carbohydrate content.

In Fig. 4 the reciprocals of sedimentation coefficients of the slow components were plotted against concentration (Fig. 3) and extrapolated to infinite dilution by the guidance of the linear regression line to yield $30.2 \mathrm{~S}$. It should be noted that the regression line was obtained by combined use of the data of thick (closed circle) and thin (open circle) white ovomucins since dispersion of the data is large enough to outweigh significant differences, 


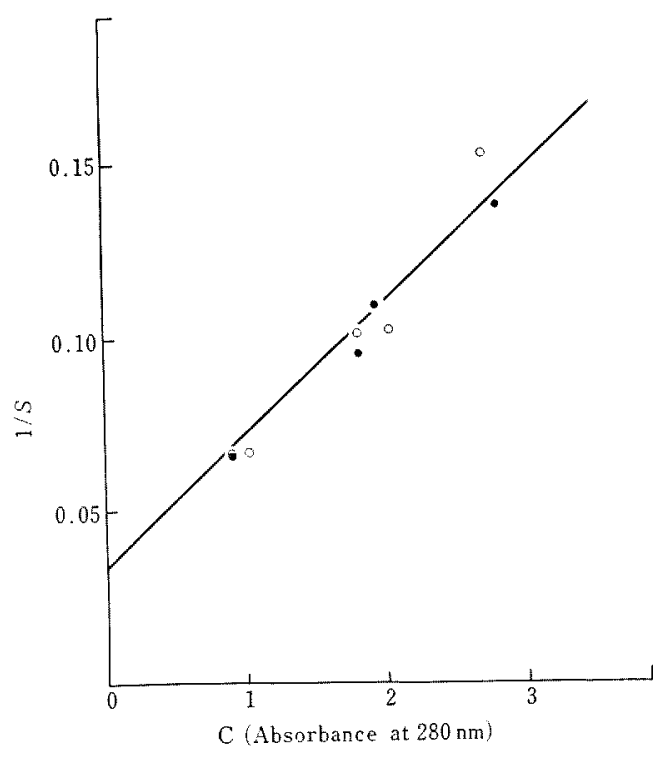

FIG. 4. The Concentration Dependence of Sedimentation Coefficients of the Slow Ovomucin Components.

- and $O$, the slow components of thick and thin white fractions respectively; the concentrations are given in terms of absorbance at $280 \mathrm{~nm}$.

if any, between thick and thin white ovomucins. Scattering of the apparent sedimentation coefficients often occurs in the velocity sedimentation of mixtures, where pronounced Johnston-Ogston effect operates. Sedimentation coefficient of the fast sedimenting ovomucin component similarly obtained was $35.0 \mathrm{~S}$. Again, the data of thick and thin white ovomucins were combined to evaluate sedimentation coefficient at infinite dilution (Fig. 5). It should be noted that the sedimentation coefficients of the slow and fast ovomucin components, $30.2 \mathrm{~S}$ and $35.0 \mathrm{~S}$ respectively, are not corrected to the standard condition.

The chemical composition of the ovomucin fractions is given in Table I. It appears that the carbohydrate content of thick white ovomucin is somewhat higher than that of thin white ovomucin. This could only be attributed to difference in the relative ratio of the slow and fast components between thick and thin white ovomucins.

In Table II was presented the amino acid composition of thick and thin white ovomu-

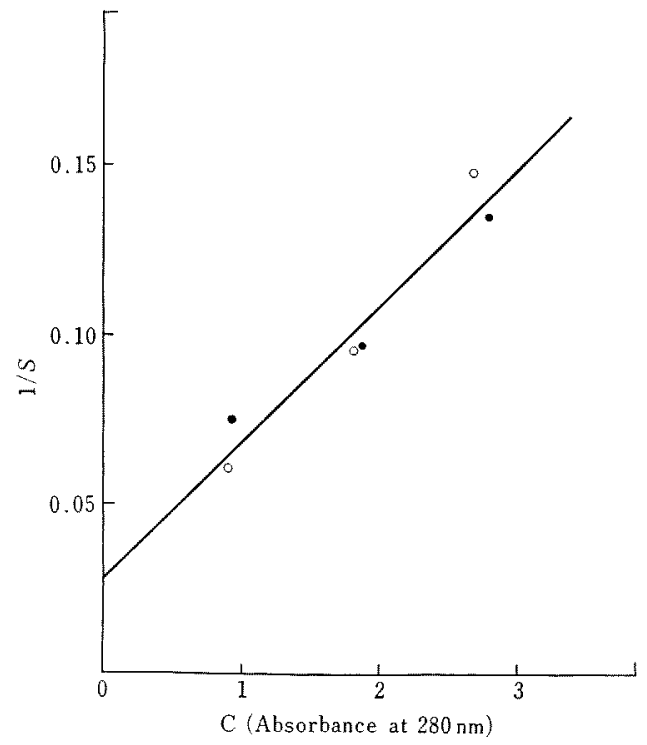

FIG. 5. The Concentration Dependence of Sedimentation Coefficients of the Fast Ovomucin Components.

- and $O$, the fast components of thick and thin white fractions respectively; the concentrations are given in terms of absorbance at $280 \mathrm{~nm}$.

Table I. Chemtcal Composition of the Ovomucins ISOLATED FROM THICK WHITE (a) AND THIN WhITE (b) (g/100 g)

\begin{tabular}{lrr}
\hline & (a) & (b) \\
\hline Protein & 66.1 & 71.5 \\
Hexose & 11.4 & 9.7 \\
Hexosamine & 12.1 & 10.6 \\
Galactosamine & 4.1 & 2.9 \\
Sialic acid & 7.4 & 6.0 \\
\hline
\end{tabular}

cins. No appreciable difference was found in the composition of the two ovomucin fractions, a result confirming general similarity of the two ovomucin fractions. It should be added that the amino acid composition given in Table II is in good agreement with the reported profiles for the ovomucin prepared from thick white $^{6)}$ or whole white ${ }^{8,21)}$ by the method of Brooks and Hale. ${ }^{2)}$

\section{DISCUSSION}

So far as seen from the limited data presented here, undisputed differences between thick and thin white ovomucins are i) that the former 
Table II. Amino Acid Composition of the Ovomucins Isolated FROM THICK White (a) AND THIN WhITE (b) (number of residues/100 amino acid residues)

\begin{tabular}{lrrrrrrr} 
& (a) & (b) & & & (a) & (b) \\
\hline Aspartic acid & 11.11 & 9.50 & & Methionine & 1.62 & 1.72 \\
Threonine & 638 & & 8.74 & & Isoleucine & 5.56 & 5.14 \\
Serine & 901 & 9.65 & & Leucine & 6.14 & 7.47 \\
Glutamic acid & 917 & 9.75 & & Tyrosine & 3.33 & 3.42 \\
Proline & 223 & 2.25 & & Phenylalanine & 4.98 & 4.95 \\
Glycine & 8.38 & 863 & & Lysine & 6.45 & 5.41 \\
Alanine & 6.05 & 6.56 & & Histidine & 1.90 & 2.19 \\
Valine & 7.56 & 7.04 & & Arginine & 3.35 & 3.29 \\
Cystine(half) & 6.72 & 6.30 & & & & \\
\hline
\end{tabular}

exists in more concentrated form and, with certain uncertainty, ii) that the carbohydrate content of the former is somewhat higher than that of the latter. Although it may be true that ovomucin palys a decisive role in maintaining the gel-like properties of thick white, ${ }^{2,22}$ the two items mentioned above appear to be insufficient to account for the vast changes that follow after natural liquefaction. Since sedimentation coefficient of ovomucin appears to remain unchanged before and after liquefaction, it is unlikely that the liquefaction process involves chemical degradation of ovomucin, although some minor raptures of glycosidic linkages of oligosaccharide chains may occur.

It seems that the mechanism for thinning of the thick white is associated with physical deterioration of certain ordered network of ovomucin maintained by hydrogen bonding formation, hydrophobic interaction, etc. Susceptibility of the gel structure of thick white to SDS and rapid solubilization of thin white over thick white may be better interpreted by the use of the postulated model. Brooks and Hale ${ }^{22}$ reported that ovomucinlysozyme complex is responsible for the gellike structure of thick white and Kato et al. ${ }^{9}$ ) recently proposed reductive degradation model of ovomucin-lysozyme network to explain the liquefaction process. However, extensive investigations are yet needed before this problem is conclusively elucidated.

\section{REFERENCES}

1) A. Eichholz, J. Physiol. (London), 23, 163 (1898).

2) J. Brooks and H. P. Hale, Biochim. Biophys. Acta, 32, 237 (1959).

3) A. Gottschalk and P. E. Lind, Brit. J. Exptl. Pathol., 30, 85 (1959).

4) F. Lanni, D. G. Sharp, E. A. Eckert, E. S. Dillon, D. Beard and J. W. Beard, J. Biol. Chem., 179, 1275 (1949).

5) D.S. Robinson and J. B. Monsey, Biochim. Biophys. Acta, 83, 368 (1964).

6) D. S. Robinson and J. B. Monsey, Biochem. J., 121, 537 (1971).

7) D. S. Robinson and J. B. Monsey, ibid., 100, 61 P (1966).

8) J. W. Donovan, J. G. Davis and L. M. White, Biochim. Biophys. Acta, 207, 190 (1970).

9) A. Kato, R. Nakamura and Y. Sato, Agr. Biol. Chem., 35, 351 (1971).

10) R. Nakamura, K. Yoshikawa and Y. Sato, Nippon Nogeikagaku Kaishi, 35, 636 (1961).

11) D.S. Robinson and J. B. Monsey, Biochem. J., 115, 64 P (1969).

12) A. L. Shapiro, E. Viñuela and J. V. Maizel, Jr., Biochem. Biophys. Res. Commun., 28, 815 (1967).

13) G. Fairbanks, T. L. Steck and D. F. H. Wallach, Biochemistry, 10, 2606 (1971).

14) D. Aminoff, Biochem. J., 81, 384 (1961).

15) C. Cessi and F. Piliego, ibid., 77, 508 (1960).

16) C. Cessi and F. Serafini-Cessi, ibid., 88, 132 (1963).

17) M. Dubois, K. A. Gilles, J. K. Hamilton, P. A. Rebers and F. Smith, Anal. Chem., 28, 350 (1956).

18) J. P. Johnston and A. G. Ogston, Trans. Faraday Soc., 42, 789 (1946).

19) J. W. Gofman, F. T. Lindgren and H. Elliot, J. Biol. Chem., 179, 973 (1949).

20) M. Janado and T. Nishida, Agr. Biol. Chem., 31, 802 (1967).

21) D. T. Osuga and R. E. Feeney, Arch. Biochem. Biophys., 124, 560 (1968).

22) J. Brooks and H. P. Hale, Biochim. Biophys. Acta, 46, 289 (1961). 